\title{
Vietoris-Rips Complexes of Planar Point Sets
}

\author{
Erin W. Chambers • Vin de Silva · Jeff Erickson • \\ Robert Ghrist
}

Received: 3 December 2008 / Revised: 29 May 2009 / Accepted: 19 June 2009 /

Published online: 8 July 2009

(C) Springer Science+Business Media, LLC 2009

\begin{abstract}
Fix a finite set of points in Euclidean $n$-space $\mathbb{E}^{n}$, thought of as a pointcloud sampling of a certain domain $D \subset \mathbb{E}^{n}$. The Vietoris-Rips complex is a combinatorial simplicial complex based on proximity of neighbors that serves as an easilycomputed but high-dimensional approximation to the homotopy type of $D$. There is a natural "shadow" projection map from the Vietoris-Rips complex to $\mathbb{E}^{n}$ that has as its image a more accurate $n$-dimensional approximation to the homotopy type of $D$.

We demonstrate that this projection map is 1-connected for the planar case $n=2$. That is, for planar domains, the Vietoris-Rips complex accurately captures connectivity and fundamental group data. This implies that the fundamental group of a Vietoris-Rips complex for a planar point set is a free group. We show that, in contrast, introducing even a small amount of uncertainty in proximity detection leads to "quasi"-Vietoris-Rips complexes with nearly arbitrary fundamental groups. This
\end{abstract}

EWC supported by NSF MSPA-MCS \# 0528086.

VdS supported by DARPA SPA \# 30759.

JE supported by NSF MSPA-MCS \# 0528086.

RG supported by DARPA SToMP \# HR0011-07-1-0002 and NSF MSPA-MCS \# 0528086.

E.W. Chambers $(\bowtie)$

Department of Mathematics and Computer Science, St. Louis University, St. Louis, USA

e-mail: echambe5@slu.edu

\section{V. de Silva}

Department of Mathematics, Pomona College, Claremont, USA

e-mail: vin.desilva@pomona.edu

J. Erickson

Department of Computer Science, University of Illinois, Urbana-Champaign, USA

e-mail: jeffe@cs.uiuc.edu

R. Ghrist

Departments of Mathematics and Electrical/Systems Engineering, University of Pennsylvania, Philadelphia, USA

e-mail: ghrist@math.upenn.edu 
topological noise can be mitigated by examining a pair of quasi-Vietoris-Rips complexes and using ideas from persistent topology. Finally, we show that the projection map does not preserve higher-order topological data for planar sets, nor does it preserve fundamental group data for point sets in dimension larger than three.

Keywords Topology $\cdot$ Rips complex $\cdot$ Quasi-Rips complex

\section{Introduction}

Given a set $X$ of points in Euclidean space $\mathbb{E}^{n}$, the Vietoris-Rips complex $\mathcal{R}_{\epsilon}(X)$ is the abstract simplicial complex whose $k$-simplices are determined by subsets of $k+1$ points in $X$ with diameter at most $\epsilon$. For simplicity, we set $\epsilon=1$ and write $\mathcal{R}:=\mathcal{R}_{1}(X)$ for the remainder of the paper, with the exception of Sect. 4. For brevity, we often refer to $\mathcal{R}$ as the Rips complex. The Rips complex is an example of a flag completion - the maximal simplicial complex with a given 1-skeleton.

The Vietoris-Rips complex was used by Vietoris [19] in the early days of homology theory as a means of creating finite simplicial models of metric spaces. Within the past two decades, Rips' reinvention of the concept has been utilized frequently in geometric group theory [13] as a means of building simplicial models for group actions. Most recently, Vietoris-Rips complexes have been used heavily in computational topology, as a simplicial model for point-cloud data [3-5, 8], and as simplicial completions of communication links in sensor networks [9, 10, 17].

The utility of Rips complexes in computational topology is as an easily computed and stored approximation to the topology of a cloud of points. We make this notion more specific. To a collection of points, one can assign a different simplicial model called the Čech complex that accurately captures the homotopy type of the cover of these points by balls. Formally, given a set $X$ of points in some Euclidean space $\mathbb{E}^{n}$, the $\check{C}_{\text {ech }}$ complex $\mathcal{C}_{\epsilon}(X)$ is the abstract simplicial complex where a subset of $k+1$ points in $X$ determines a $k$-simplex if and only if they lie in a ball of radius $\epsilon / 2$. The Čech complex is equivalently the nerve of the set of closed balls of radius $\epsilon / 2$ centered at points in $X$. The Čech theorem (or Nerve lemma, see, e.g., [2]) states that $\mathcal{C}_{\epsilon}(X)$ has the homotopy type of the union of these balls. Thus, the Cech complex is an appropriate simplicial model for the topology of the point cloud (where the parameter $\epsilon$ is a variable).

There is a price for the high topological fidelity of a Čech complex. Given the point set, it is nontrivial to compute and store the simplices of the Čech complex. The virtue of a Rips complex is that it is determined completely by its 1 -skeletonthe proximity graph of the points. (This is particularly useful in the setting of ad hoc wireless networks, where the hardware establishes communication links based, ideally, on proximity of nodes.) The penalty for this simplicity is that it is not immediately clear what is encoded in the homotopy type of $\mathcal{R}$. Like the Čech complex, it is not generally a subcomplex of its host Euclidean space $\mathbb{E}^{n}$, and, unlike the Čech complex, it need not behave like an $n$-dimensional space at all: $\mathcal{R}$ may have nontrivial topological invariants (homotopy or homology groups) of dimension $n$ and above. Clearly, for $\epsilon$ too small or too large, the fidelity of both Čech and Rips complexes fails. For a given $\epsilon$ and a sufficiently dense sampling of points on a manifold, both 

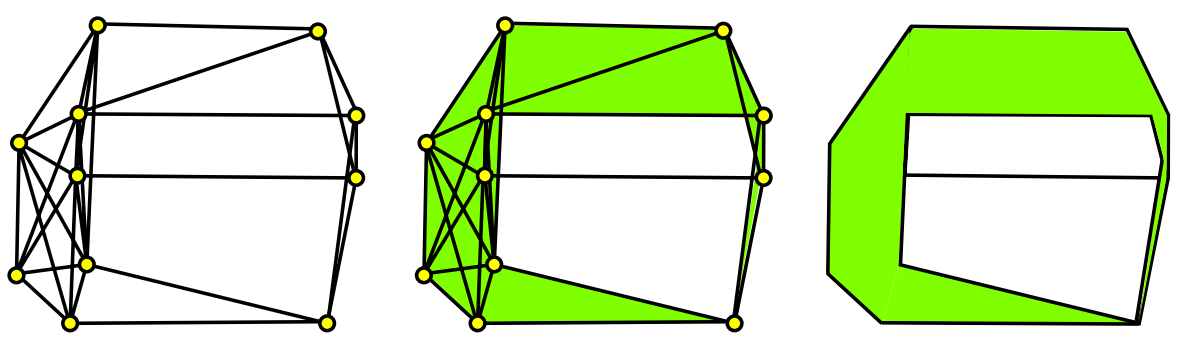

Fig. 1 A connectivity graph in the plane (left) determines a five-dimensional (Vietoris-)Rips complex (center) and its two-dimensional projected shadow (right)

the Čech and Rips complexes return topologically faithful simplicial approximations (see, e.g., [15, 18]).

The disadvantage of both Čech and Rips complexes are in their rigid cut-offs as a function of distance between points. Arbitrarily small perturbations in the locations of the points can have dramatic effects on the topology of the associated simplicial complexes. Researchers in sensor networks are acutely aware of this limitation, given the amount of uncertainty and fluctuation in wireless networks. To account for this, several researchers in sensor networks have used a notion of a distance-based communication graph with a region of uncertain edges $[1,7,16]$. This motivates the following construction.

Fix an open uncertainty interval $\left(\epsilon, \epsilon^{\prime}\right)$ which encodes connection errors as a function of distance. For all nodes of distance $\leq \epsilon$, there is an edge, and for all nodes of distance $\geq \epsilon^{\prime}$, no edge exists. For nodes of distance within $\left(\epsilon, \epsilon^{\prime}\right)$, a communication link may or may not exist. A quasi-Rips complex $\mathcal{Q}$ with uncertainty interval $\left(\epsilon, \epsilon^{\prime}\right)$ is the simplicial flag completion of such a graph. We note that this does not model temporal uncertainty, merely spatial.

A completely different model of simplicial complexes associated to a point cloud comes from considering shadows. Any abstract simplicial complex with vertices indexed by geometric points in $\mathbb{E}^{n}$ (e.g., a Rips, Čech, or quasi-Rips complex) has a canonical shadow in $\mathbb{E}^{n}$, which strikes a balance between computability and topological faithfulness. For, say, a Rips complex, the canonical projection $p: \mathcal{R} \rightarrow \mathbb{E}^{n}$ is the well-defined function that maps each simplex in $\mathcal{R}$ affinely onto the convex hull of its vertices in $\mathbb{E}^{n}$. This projection map is continuous and piecewise-linear. The shadow $\mathcal{S}$ is the image $p(\mathcal{R})$ of this projection map.

This paper studies the topological faithfulness of the projection map $p$ (see Fig. 1). Specifically, we look at the connectivity of $p$. Recall that a topological map $f: X \rightarrow Y$ is $k$-connected if the induced homomorphisms on homotopy groups $\pi_{i}(p): \pi_{i}(X) \rightarrow \pi_{i}(Y)$ are isomorphisms for all $0 \leq i \leq k:$ e.g., a 1-connected map preserves path-connectivity and fundamental group data.

We can now list the principal results of the paper, ordered as they appear in the following sections.

(1) For any set of points in $\mathbb{E}^{2}, \pi_{1}(p): \pi_{1}(\mathcal{R}) \rightarrow \pi_{1}(\mathcal{S})$ is an isomorphism.

(2) The fundamental group of any planar Rips complex is free. 
(3) Given any finitely presented group $G$, there exists a quasi-Rips complex $\mathcal{Q}$ with arbitrarily small uncertainty interval such that $\pi_{1}(\mathcal{Q})$ is a free extension of $G$.

(4) Given a pair of quasi-Rips complexes $\mathcal{Q}_{s} \subset \mathcal{Q}_{w}$ with disjoint uncertainty intervals, the rank of the homology map $H_{1}\left(\mathcal{Q}_{s}\right) \rightarrow H_{1}\left(\mathcal{Q}_{w}\right)$ induced by inclusion is bounded by the number of holes in a shadow of intermediate radius.

(5) The projection map $p$ on $\mathbb{R}^{n}$ is always $k$-connected for $k=0$ or $n=1$. For all other cases except $(k, n)=(1,2)$ and, perhaps, $(1,3), k$-connectivity fails on $\mathbb{R}^{n}$ (see Fig. 8).

Items (1), (2), and (5) above are of most interest in the classification of VietorisRips complexes. Items (2), (3), and (4) were proved in response to questions raised in sensor networks. Specifically, (2) answers the question of how well a Rips complex approximates a coverage region for an ad hoc wireless network of idealized sensors, and whether, e.g., $H_{1}$ in integer coefficients will ever return torsional elements (which impacts which coefficient groups can be safely used in computations!). Items (3) and (4) were proved as, respectively, negative and positive answers to the question of whether a quasi-Rips complex provides a useful approximation to the topology of coverage for a sensor network with uncertainty in wireless communications near the range limits.

\section{Planar Rips Complexes and Their Shadows}

In this section, we restrict attention to the two-dimensional case.

\subsection{The Shadow Complex}

The shadow $\mathcal{S}$ is a polyhedral subset of the plane. By Carathéodory's theorem [11], $\mathcal{S}$ is the projection of the 2-skeleton of $\mathcal{R}$. Since the vertices of $\mathcal{R}$ are distinct points in the plane, it follows that distinct edges of $\mathcal{R}$ have distinct images under $p$, and these are nondegenerate. Informally we will identify vertices and edges of $\mathcal{R}$ with their images under $p$. On the other hand, $p$ may be degenerate on 2-simplices.

We can canonically decompose $\mathcal{S}$ into a two-dimensional shadow complex as follows:

- A shadow vertex is either a vertex of $\mathcal{R}$ or a point of transverse intersection of two edges of $\mathcal{R}$. We write $\mathcal{S}^{(0)}$ for the set of shadow vertices.

- A shadow edge is the closure of any component of $p\left(\mathcal{R}^{(1)}\right) \backslash \mathcal{S}^{(0)}$. Each shadow edge is a maximal line segment contained in a Rips edge, with no shadow vertices in its interior. We write $\mathcal{S}^{(1)}$ for the union of all shadow vertices and shadow edges.

- Finally, a shadow face is the closure of any bounded component of $\mathbb{E}^{2} \backslash \mathcal{S}^{(1)}$.

The fundamental group $\pi_{1}(\mathcal{S})$ may now be described in terms of combinatorial paths of shadow edges modulo homotopy across shadow faces, whereas $\pi_{1}(\mathcal{R})$ may be described in terms of combinatorial paths of Rips edges modulo homotopy across Rips faces. This description opens the door to combinatorial methods in the proof that $\pi_{1}(p)$ is an isomorphism. 
Fig. 2 The last case of Proposition 2.2

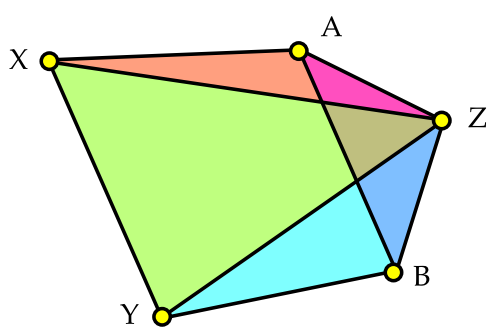

\subsection{Technical Lemmas}

Theorem 3.1 will follow from reduction to three special cases. We prove these cases in this subsection. We use the following notation. Simplices of a Rips complex will be specified by square braces, e.g., $[A B C]$. Images in the shadow complex will be denoted without adornment, e.g., $A B C$. The Euclidean length of an edge $A B$ will be denoted $|A B|$. Braces $\langle\cdot\rangle$ will be used to denote the span in $\mathcal{R}$ : the smallest subcomplex containing a given set of vertices, e.g., $\langle A B C D\rangle$.

The following propositions address the three special cases of Theorem 3.1 which are used to prove the theorem. Certain induced subcomplexes of $\mathcal{R}$ are shown to be simply connected. In the first two cases, it is helpful to establish the stronger conclusion that these subcomplexes are cones: all maximal simplices share a common vertex, called the apex. The first of these cases is trivial and well known (viz., [9, 12]).

Proposition 2.1 Let $\mathcal{R}=\langle A B Y Z\rangle$ be a Rips complex containing simplices $[A B]$ and $[Y Z]$ whose images in $\mathcal{S}$ intersect. Then $\mathcal{R}$ is a cone.

Proof Let $x$ be the common point of $A B$ and $Y Z$. Each edge is split at $x$ into two pieces, at most one of which can have length more than one-half. The triangle inequality implies that the shortest of these four half-edges must have its endpoint within unit distance of both endpoints of the traversing edge, thus yielding a 2-simplex in $\mathcal{R}$.

Proposition 2.2 Let $\mathcal{R}=\langle A B X Y Z\rangle$ be a Rips complex containing simplices $[A B]$ and $[X Y Z]$ whose images in $\mathcal{S}$ intersect. Then $\mathcal{R}$ is a cone.

Proof The edge $A B$ intersects the triangle $X Y Z$. If $A B$ intersects only one edge of $X Y Z$, then one vertex of $A B$ (say, $A$ ) lies within $X Y Z$ and cones off a 3-simplex $[A X Y Z]$ in $\mathcal{R}$. Therefore, without a loss of generality we may assume that $A B$ crosses $Z Y$ and $Z X$.

By Proposition 2.1, the subcomplexes $\langle A B X Z\rangle$ and $\langle A B Y Z\rangle$ are cones. If these two cones have the same apex, then the entire Rips complex $\mathcal{R}$ is a cone with that apex. Similarly, if either apex lies inside the image triangle $X Y Z$, then $\mathcal{R}$ is a cone with that apex. The only remaining possibility is that $A$ is the apex of one subcomplex and $B$ is the apex of the other (see Fig. 2); in this case, $\mathcal{R}$ is a cone over $Z$, since both $A$ and $B$ are connected to $Z$. 
Fig. 3 The setup for Proposition 2.3

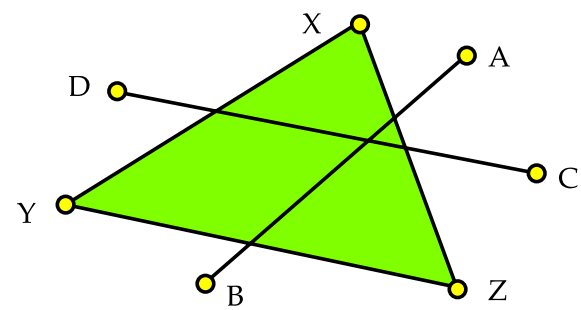

Proposition 2.3 Let $\mathcal{R}=\langle A B C D X Y Z\rangle$ be a Rips complex containing simplices $[A B],[C D]$, and $[X Y Z]$ whose images in $\mathcal{S}$ meet in a common point. Moreover, assume that none of $A, B, C, D$ lies in the interior of $X Y Z$. (See Fig. 3.) Then $\pi_{1}(\mathcal{R})$ is trivial.

To prove Proposition 2.3, we use two further geometric lemmas.

Lemma 2.4 Let $\mathcal{R}=\langle B X Y Z\rangle$ be a Rips complex containing simplex [XYZ]. If $M$ is a point in $X Y Z$ such that $|B M| \leq \frac{1}{2}$, then $\mathcal{R}$ contains at least one of the edges $[B X],[B Y],[B Z]$.

Proof If $B$ lies in $X Y Z$, then all three edges belong to $\mathcal{R}$. Otherwise, $B M$ meets the boundary of $X Y Z$ at a point $M^{\prime}$. We may assume that $M^{\prime}$ lies on $X Y$, with $\left|M^{\prime} X\right| \leq\left|M^{\prime} Y\right|$. Then $|B X| \leq\left|B M^{\prime}\right|+\left|M^{\prime} X\right| \leq \frac{1}{2}+\frac{1}{2}=1$.

Lemma 2.5 Let $\mathcal{R}=\langle A B C X Y Z\rangle$ be a Rips complex containing simplices $[A B C]$ and $[X Y Z]$. Suppose that $A B$ intersects $X Y Z$ but $B C$ and $A C$ do not. Then $\mathcal{R}$ is a cone.

Proof The hypotheses of the lemma imply that at least one of the points $X, Y$, or $Z$ lies in the interior of $A B C . \mathcal{R}$ is a cone on this point.

Proof of Proposition 2.3 We argue by exhaustive case analysis that $\mathcal{R}$ contains no minimal noncontractible cycle.

Suppose $\gamma$ is a minimal noncontractible cycle in $\mathcal{R}$. Because $\mathcal{R}$ is a flag completion, $\gamma$ must consist of at least four Rips edges. Our previous propositions imply that this cycle intersects each simplex $[A B],[C D]$, and $[X Y Z]$ at least once. By minimality, $\gamma$ contains at most one edge of $[X Y Z]$. Thus, we may assume without loss of generality (by relabeling if necessary) that $\gamma$ is of the form $A(B) C(D) X(Y)$ where (.) denotes an optional letter.

Claim 1: In a minimal cycle, the subwords $A B C D, C D X Y, X Y A B$ are impossible. Proposition 2.1 (in the first case) and Proposition 2.2 (in the last two cases) imply that the subpaths corresponding to these subwords are homotopic (relative to endpoints) within a cone subcomplex to a path with at most two edges, contradicting the minimality of $\gamma$.

Claim 1 implies that there is at most (i.e., exactly) one optional letter. This leaves three possible minimal noncontractible cycles: $A C X Y, A B C X$, and $A C D X$. The 

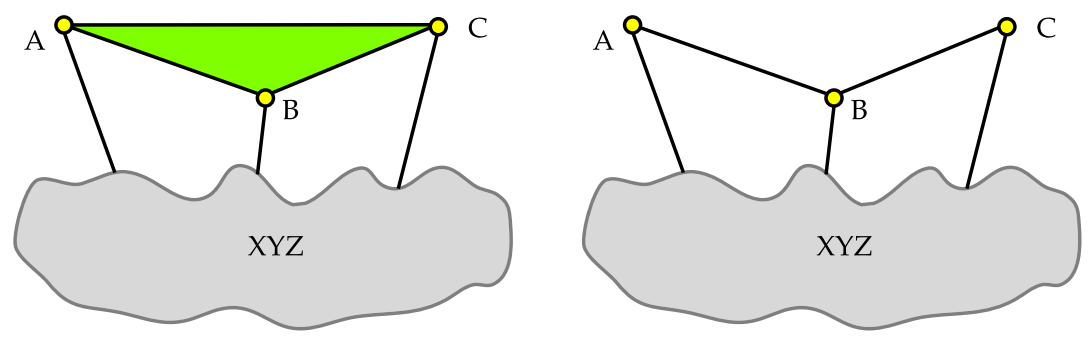

Fig. $4 A C X Y$ (left), or $A B C X$ (right), splits into two cycles in the presence of $[B X],[B Y]$, or $[B Z]$

last two cases differ only by relabeling, so it suffices to consider only $A C X Y$ and $A B C X$.

Claim 2: $A C X Y$ is impossible. Suppose $A C X Y$ is a cycle in $\mathcal{R}$. If $A C$ meets $X Y Z$, then Proposition 2.2 implies that $\langle A C X Y Z\rangle$ is a cone, so $A C X Y$ is contractible. Thus, we can assume that $A C$ does not meet $X Y Z$.

By Proposition 2.1, either $[B C]$ or $[A D]$ is a Rips edge. Without loss of generality, assume that $[B C]$ is a Rips edge; then $[A B C]$ is a Rips triangle. If $B C$ does not meet $X Y Z$, then Lemma 2.5 implies that $\langle A B C X Y Z\rangle$ is a cone, and hence that $A C X Y$ is contractible. Thus we can assume that $B C$ intersects $X Y Z$.

Proposition 2.2 now implies that both $\langle A B X Y Z\rangle$ and $\langle B C X Y Z\rangle$ are cones. If any of the segments $[B X],[B Y],[B Z]$ is a Rips edge, then the cycle $A C X Y$ is homotopic to the sum of two cycles, contained respectively in the cones $\langle A B X Y Z\rangle$ and $\langle B C X Y Z\rangle$, and hence is contractible. See Fig. 4(a).

We can therefore assume that none of the segments $[B X],[B Y],[B Z]$ is a Rips edge. In this case, the apex of $\langle A B X Y Z\rangle$ must be $A$. In particular, the diagonal $[A X]$ of the cycle $A C X Y$ belongs to $\mathcal{R}$, and so $A C X Y$ is contractible. This completes the proof of Claim 2.

Claim 3: $A B C X$ is impossible. Suppose $A B C X$ is a cycle in $\mathcal{R}$. If either $[A C]$ or $[B X]$ is a Rips edge, then $A B C X$ is trivially contractible. Moreover, if either $[B Y]$ or $[B Z]$ is a Rips edge, then the cycle $A B C X$ reduces to the sum of two cycles, as in Fig. 4(b). The left cycle is contractible by Proposition 2.2, and the right cycle is contractible by Claim 2 (suitably relabeled), so $A B C X$ is contractible in that case too. We can therefore assume that none of the segments $[A C],[B X],[B Y]$, or $[B Z]$ is a Rips edge.

Now let $M$ be a common point of intersection of $A B, C D$, and $X Y Z$. Lemma 2.4 implies that $|B M|>\frac{1}{2}$, and so $|A M|=|A B|-|B M| \leq \frac{1}{2}$. Since $|A C|>1$, we have $|C M|=|A C|-|A M|>\frac{1}{2}$, and so $|D M|=|C D|-|C M| \leq \frac{1}{2}$. These inequalities imply that $|A D| \leq|A M|+|D M| \leq 1$, so $[A D]$ is a Rips edge.

It follows that $\mathcal{R}$ contains the cycle $A D C X$. This cycle is homotopic to $A B C X$, since $\langle A B C D\rangle$ is a cone by Proposition 2.1. Lemma 2.4 implies that at least one of the segments $[D X],[D Y],[D Z]$ must be a Rips edge. Arguing as before, with $D$ in place of $B$, we conclude that $A D C X$, and thus $A B C X$, is contractible. This completes the proof of Claim 3. 


\subsection{Lifting Paths Via Chaining}

For any path $\alpha$ in $\mathcal{R}^{(1)}$, the projection $p(\alpha)$ is a path in $\mathcal{S}^{(1)}$, but not every shadow path is the projection of a Rips path. Every oriented shadow edge in $\mathcal{S}$ is covered by one or more oriented edges in $\mathcal{R}$. Thus to every path in $\mathcal{S}^{(1)}$ can be associated a sequence of oriented edges in $\mathcal{R}$. These edges do not necessarily form a path, but projections of consecutive Rips edges necessarily intersect at a shadow vertex.

Definition 2.6 Let $[A B]$ and $[C D]$ be oriented Rips edges induced by consecutive edges in some shadow path. A chaining sequence is a path from $A$ to $D$ in the subcomplex $\langle A B C D\rangle$ which begins with the edge $A B$ and ends with the edge $C D$.

If we concatenate chaining sequences of shadow edges in $\mathcal{S}$ by identifying the Rips edges in the beginning and end of adjacent lifting sequences, we obtain a lift of the shadow path to $\mathcal{R}$. For any shadow path $\alpha$ in $\mathcal{S}$, we let $\widehat{\alpha}$ denote a lift of $\alpha$ to the Rips complex by means of chaining sequences. Note that the lift of a shadow path is not a true lift with respect to the projection map $p$ - the endpoints, for example, may differ.

Lemma 2.7 For any path $\alpha$ in $\mathcal{S}^{(1)}$, any two lifts of $\alpha$ to $\mathcal{R}$ with the same endpoints are homotopic in $\mathcal{R}$ rel endpoints.

Proof Let $\sigma$ and $\tau$ be consecutive shadow edges in $\alpha$, and let $[A B]$ and $[C D]$ be Rips edges such that $\sigma \subseteq A B$ and $\tau \subseteq C D$. Proposition 2.1 implies that all chaining sequences from $A$ to $D$ are homotopic rel endpoints in $\langle A B C D\rangle$, and thus in $\mathcal{R}$. If every shadow edge in $\alpha$ lifts to a unique Rips edge, the proof is complete.

On the other hand, suppose $\tau \subseteq C D \cap C^{\prime} D^{\prime}$ for some Rips edge $\left[C^{\prime} D^{\prime}\right]$ that overlaps $[C D]$. Proposition 2.1 implies that both $\left[C C^{\prime}\right]$ and $\left[D D^{\prime}\right]$ are Rips edges. Moreover, since $A B$ intersects $C D \cap C^{\prime} D^{\prime}$, any chaining sequence from $A$ to $D$ is homotopic rel endpoints in $\mathcal{R}$ to any chaining sequence from $A$ to $D^{\prime}$ followed by $\left[D^{\prime} D\right]$. Thus, concatenation of chaining sequences is not dependent on uniqueness of edge lifts.

We next show that the projection of a lift of any two consecutive shadow edges is homotopic to the original edges.

Lemma 2.8 For any two adjacent shadow edges $w x$ and $x y$, where $A B$ and $C D$ are Rips edges with $w x \subseteq A B$ and $x y \subseteq C D, p(\widehat{w x \cdot x y})$ is homotopic rel endpoints to the path $A B x C D$ in $\mathcal{S}$.

Proof See Fig. 5 for setup. Consider the possible chaining sequences from $A$ to $D$ for $w x \cdot x y$. Either $B C$ or $A D$ must exist in $\mathcal{R}$ by Proposition 2.1 .

Suppose $B C$ exists. By Lemma 2.7, the chaining sequence is the Rips path $A B C D$ (up to homotopy rel endpoints). Either the triangle $[A B C]$ or the triangle $[B C D]$ exists in $\mathcal{R}$ by Proposition 2.1 , so the triangle $B C x$ is in shadow. This gives that $A B C D \simeq A x D \simeq A B x C D$ in $\mathcal{S}$. 
Fig. 5 The setting for Lemma 2.8

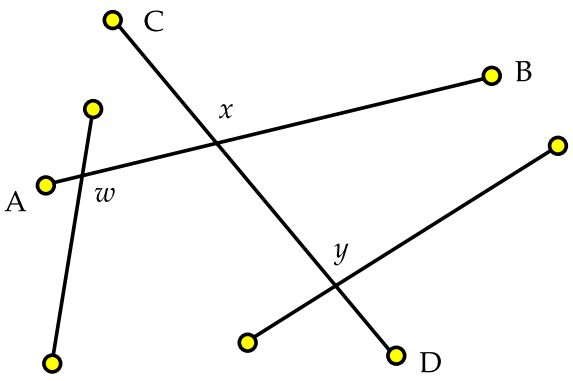

If $B C$ is not a Rips edge, then $A D$ must be a Rips edge. By Lemma 2.7, the chaining sequence is the Rips path $A B A D C D$ (up to homotopy rel endpoints). Either the triangle $[A C D]$ or the triangle $[A B D]$ exists in $\mathcal{R}$ by Proposition 2.1. Therefore, $A D x$ lies in the shadow, so we get $A B A D C D \simeq A B x C D$ in $\mathcal{S}$.

Lemma 2.9 For any lift $\widehat{\alpha}$ of any shadow path $\alpha$ with endpoints in $p\left(\mathcal{R}^{(0)}\right)$, we have $p(\widehat{\alpha}) \simeq \alpha$ rel endpoints.

Proof For each pair of consecutive shadow edges $w x$ and $x y$ in $\alpha$, where $w x \subseteq A B$, $x y \subseteq C D$, and $A B$ and $C D$ are Rips edges, Lemma 2.8 says that the projection of their lifting sequence deforms back to $A B x C D$. Every adjacent pair of chaining sequences can still be identified along common edges, since each ends with the first edge in the next one along $\alpha$. The projection is homotopic rel endpoints to the original path $\alpha$ except for spikes of the form $x B$ and $x C$ at each shadow junction, which can be deformation retracted, giving $p(\widehat{\alpha}) \simeq \alpha$.

\section{1-connectivity on $\mathbb{R}^{2}$}

The following is the main theorem of this paper.

Theorem 3.1 For any set of points in $\mathbb{E}^{2}, \pi_{1}(p): \pi_{1}(\mathcal{R}) \rightarrow \pi_{1}(\mathcal{S})$ is an isomorphism.

Proof Assume that all $\pi_{1}$ computations are performed with a basepoint in $p\left(\mathcal{R}^{(0)}\right)$, to remove ambiguity of endpoints in lifts of shadow paths to $\mathcal{R}$. Surjectivity of $p$ on $\pi_{1}$ follows from Lemma 2.9 and the fact that any loop in $\mathcal{S}$ is homotopic to a loop of shadow edges thanks to the cell structure of $\mathcal{S}$.

To prove injectivity, note that any contractible cycle in $\mathcal{S}$ is expressible as a concatenation of boundary loops of shadow faces (conjugated to the basepoint). Thanks to Lemma 2.9, injectivity of $\pi_{1}(p)$ will follow by showing that the boundary of any shadow face lifts to a contractible loop in $\mathcal{R}$. Consider therefore a shadow face $\Psi$ contained in the projection of a Rips 2-simplex [XYZ], and choose [XYZ] to be minimal in the partial order of such 2 -simplices generated by inclusion on the projections.

Write $\partial \Psi$ as $\alpha_{1} \cdot \alpha_{2} \cdots \alpha_{n}$, where the $\alpha_{i}$ are the shadow edges, and let $\left[A_{i} B_{i}\right]$ be a sequence of directed Rips edges with $\alpha_{i} \subseteq\left[A_{i} B_{i}\right]$. Neither the $A_{i}$ nor the $B_{i}$ project 
Fig. 6 The boundary of a shadow face $\Psi$ within $X Y Z$ is determined by Rips edges $\left[A_{i} B_{i}\right]$ whose projected endpoints lie outside $X Y Z$

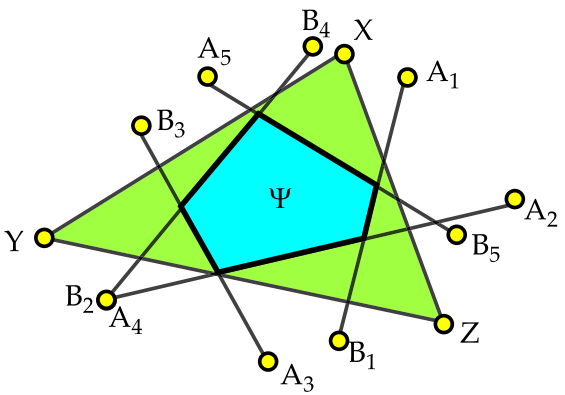

to the interior of $X Y Z$ (see Fig. 6); if any Rips vertex $W$ did so, the edges $[X W]$, $[Y W]$, and $[Z W]$ would exist in $\mathcal{R}$. As $\Psi$ cannot be split by the image of any of these three edges, it must be contained in the projected image of a Rips 2-simplex, say $[X Y W]$, whose image lies within that of $[X Y Z]$, contradicting the minimality assumption on $[X Y Z]$. The hypotheses of Proposition 2.3 thus apply to $[X Y Z]$ and the consecutive edges $\left[A_{i} B_{i}\right],\left[A_{i+1} B_{i+1}\right]$, and each complex $\left\langle A_{i} B_{i} A_{i+1} B_{i+1} X Y Z\right\rangle$ is simply connected.

Fix the vertex $X$ as a basepoint and fix a sequence of edge paths $\beta_{i}$ in $\left\langle A_{i} B_{i} X Y Z\right\rangle$ from $X$ to $A_{i}$. Such paths exist and are unique up to homotopy since (by Proposition 2.2) $\left\langle A_{i} B_{i} X Y Z\right\rangle$ is a cone. We decompose $\partial \Psi$ into loops $\gamma_{1} \cdots \gamma_{n}$, where $\gamma_{i}$ is the loop with basepoint $X$ given by

$$
\gamma_{i}=\beta_{i} \cdot\left(\widehat{\alpha_{i} \cdot \alpha_{i+1}}\right) \cdot\left[B_{i+1} A_{i+1}\right] \cdot \beta_{i+1}^{-1},
$$

where all indices are computed modulo $n$. By Proposition 2.3, each of these loops $\gamma_{i}$ is contractible; hence, so is $\widehat{\Psi}$.

Corollary 3.2 The fundamental group of a Rips complex of a planar point set is free.

\section{Quasi Rips Complexes and Shadows}

We observe that Theorem 3.1 fails for quasi-Rips complexes, even for those with arbitrarily small uncertainty intervals. The failure of Proposition 2.1 in the quasi-Rips case makes it a simple exercise for the reader to generate examples of a quasi-Rips complexes which are simply-connected but whose shadows are not. Worse failure than this is possible.

Theorem 4.1 Given any uncertainty interval $\left(\epsilon, \epsilon^{\prime}\right)$ and any finitely presented group $G$, there exists a quasi-Rips complex $\mathcal{Q}$ with $\pi_{1}(\mathcal{Q}) \cong G * F$, where $F$ is a free group.

Proof It is well known that any finitely presented group $G$ can be realized as the fundamental group of a 2-dimensional cell complex whose 0 -skeleton is a single point; whose 1-skeleton is a wedge (or "bouquet") of loops corresponding to the generators; and whose 2-cells are glued along the 1-skeleton corresponding to relations. Such a complex can be triangulated and, after a barycentric subdivision, can be assumed 

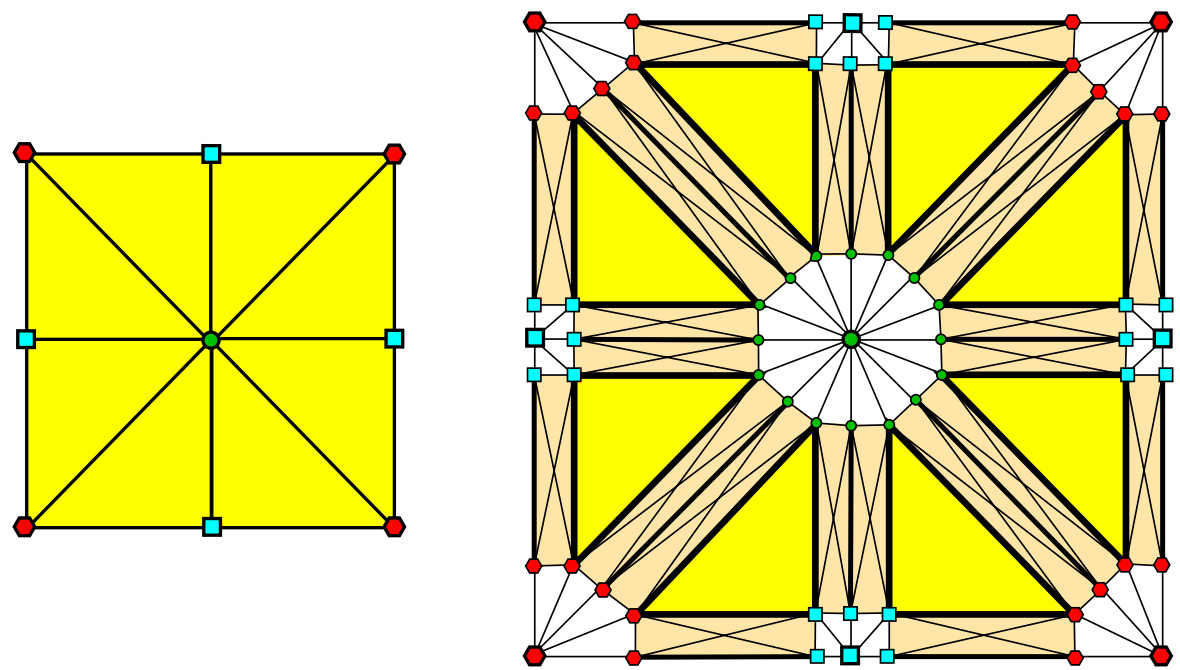

Fig. 7 A 3-colored simplicial complex $K$ and its blowup $\tilde{K}$, whose flag completion is homotopy equivalent to $K$. Opposite edges of $K$ (and thus $\tilde{K}$ ) can be identified to yield a torus, projective plane, or Klein bottle

to be 3-colored: that is, the vertex is labeled by the set $\{0,1,2\}$, and there are no edges between vertices of the same color. Call this vertex 3-colored 2-d simplicial complex $K$.

We perform a "blowup" of the complex $K$ to a 3-d simplicial complex $\tilde{K}$ as follows (see Fig. 7 for an example). Recall that the geometric realization of $K$ can be expressed as the disjoint union of closed $i$-simplices $(i=0,1,2)$ with all faces attached via simplicial gluing maps (the $\Delta$-complex [14]). To form $\tilde{K}$, take the disjoint union of closed $i$-simplices of $K$ and instead of simplicial gluing maps, use the join to connect all faces. The 3-coloring of the vertex set of $K$ is inherited by that of $\tilde{K}$.

Claim: The flag completion $\mathcal{F} \tilde{K}$ of $\tilde{K}$ is homotopic to $K$.

There is a natural collapsing map $c: \tilde{K} \rightarrow K$ which collapses the joins to simplicial identification maps. The inverse image of any point $p$ in an open 2-simplex of $K$ is the same point in the corresponding open 2 -simplex of $\tilde{K}$. The inverse image of any point $p$ in an open 1-simplex of $K$ is homeomorphic to the wedge product of closed rectangles, one for each point in the link of $p$ in $K$. The inverse image of a 0 -simplex $p \in K$ consists of the 1 -skeleton of the link of $p$ in $K$. If we complete $\tilde{K}$ to the flag completion $\mathcal{F} \tilde{K}$, then $c^{-1}(p)$ is homeomorphic to the star of $p$ in $K$. Thus, after extending the map $c$ to the flag completion of $\tilde{K}$, all fibers of $c$ are contractible, and the flag completion of $\tilde{K}$ is thus homotopic to $K$.

We now embed $\mathcal{F} \tilde{K}$ in a quasi-Rips complex $\mathcal{Q}$. Define the vertices of $\mathcal{Q}$ in $\mathbb{R}^{2}$ as follows. Fix an equilateral triangle of side length $\left(\epsilon+\epsilon^{\prime}\right) / 2$ in $\mathbb{R}^{2}$. Embed the vertices of $\tilde{K}$ arbitrarily in sufficiently small open balls (of radii no larger than $\left(\epsilon^{\prime}-\epsilon\right) / 4$ ) centered at the vertices of this triangle, respecting the 3 -coloring. For this vertex set in $\mathbb{R}^{2}$, we define $\mathcal{Q}$ by placing an edge between vertices according to the edges of $\tilde{K}$, using the fact that any two vertices not of the same color are separated by a distance within the uncertainty interval. Of course, we must also add edges between like- 
colored vertices, forming three (large) complete connected subgraphs, since these lie within the small balls. The quasi-Rips complex $\mathcal{Q}$ is the flag completion of this graph, which contains an embedded copy of $\mathcal{F} \tilde{K}$.

We determine $\pi_{1}(\mathcal{Q})$ in two steps. First, consider the addition of monochromatic edges for which the resulting flag completion has new 2-simplices which are not monochromatic.

Claim: The addition of such edges leaves $\pi_{1}$ of the flag completion unchanged. Any added monochromatic edge $e$ which creates a nonmonochromatic 2-simplex $\sigma$ must have $c(e)$ a 0 -simplex of $K$ and $c(\sigma)$ a 1-simplex of $K$, giving rise (upon taking a flag completion) to a new 3 -simplex attached along a contractible subcomplex of its boundary. The addition of all such edges leaves $\pi_{1}$ of the resulting flag completion unchanged, since all the added edges are pairwise disjoint and lead to the pairwise disjoint 3-simplices in the flag completion, and since each attaching map glues along a contractible set.

It remains to attach all edges which lead to monochromatic simplices in the flag completion.

Claim: $\pi_{1}(\mathcal{Q})$ is a free extension of $\pi_{1}(\mathcal{F} \tilde{K})$. Work one color at a time. The addition of the monochromatic simplices used to form $\mathcal{Q}$ from the resulting complex of the previous claim leads to a potentially very high-dimensional complex; nevertheless, this resulting complex is homotopic to adding an abstract colored vertex and "coning off" the flag subcomplex defined by that color. Since the flag subcomplex defined by a given color is a disjoint union of contractible sets (roughly speaking, filled-in "blowups" of vertices of $K$ ), the resulting space is homotopic to taking a wedge with (potentially many) circles and thus yields a free extension of the fundamental group of the flag completion $\mathcal{F} \tilde{K}$. This ends the claim and the proof, since $\pi_{1}(\mathcal{F} \tilde{K}) \cong \pi_{1}(K) \cong G$.

We note that the construction above may be modified so that the lower-bound Rips complex $\mathcal{R}_{\epsilon}$ is connected. If necessary, the complex can be so constructed that the inclusion map $\mathcal{R}_{\epsilon} \hookrightarrow \mathcal{R}_{\epsilon^{\prime}}$ induces an isomorphism on $\pi_{1}$ (which factors through $\left.\pi_{1}(\mathcal{Q})\right)$.

Theorem 4.1 would appear to be a cause for despair, especially for applications to sensor networks, in which the rigid unit-disc graph assumption is unrealistic. There are several works in the theoretical sensor networks literature $[1,7,16]$ that use a quasi-unit disc graph assumption. From the point of view of representing the topology of the network, this would appear to be suboptimal.

However, Theorem 3.1 is not without utility, even when only quasi-Rips complexes are available. We proceed using the principle of topological persistence: examining the topology of a map between spaces as opposed to a space alone. Specific to this case, given an inclusion $\iota: A \hookrightarrow X$ of $A \subset X$, one writes $H_{*}(A \rightarrow X)$ as the image of the induced map $H_{*}(\iota): H_{*}(A) \rightarrow H_{*}(X)$. We consider a sensor network whose communications processing can distinguish between very close nodes (via a strong signal) as opposed to moderately close nodes (via a weak signal), cf. [10]. Assuming some uncertainty in the communication ranges, one has a pair of quasi-Rips complexes $\mathcal{Q} \subset \mathcal{Q}^{\prime}$. Although the fundamental groups (and, via abelianization, $H_{1}$ ) of these quasi-Rips complexes can be uncorrelated with the topology of their shad- 
ows, their persistent homology can be used to infer genuine holes in coverage, as follows:

Proposition 4.2 Let $\mathcal{Q}_{s} \subset \mathcal{Q}_{w}$ denote two quasi-Rips complexes with disjoint uncertainty intervals $\left(\epsilon_{s}, \epsilon_{s}^{\prime}\right)$ and $\left(\epsilon_{w}, \epsilon_{w}^{\prime}\right)$. Then, in homology with field coefficients, the rank of the map $H_{1}\left(\mathcal{Q}_{s}\right) \rightarrow H_{1}\left(\mathcal{Q}_{w}\right)$ is bounded above and below:

$$
\operatorname{rank}\left(H_{1}\left(\mathcal{S}_{\epsilon_{s}} \rightarrow \mathcal{S}_{\epsilon_{w}^{\prime}}\right)\right) \leq \operatorname{rank}\left(H_{1}\left(\mathcal{Q}_{s} \rightarrow \mathcal{Q}_{w}\right)\right) \leq \operatorname{dim}\left(H_{1}\left(\mathcal{S}_{\eta}\right)\right)
$$

for any $\epsilon_{s}^{\prime} \leq \eta \leq \epsilon_{w}$.

Roughly speaking, this result says that a pair of quasi-Rips complexes, graded according to sufficiently distinct strong and weak signal links, suffices to induce information about related shadow complexes.

Proof The inclusions $\mathcal{R}_{\epsilon_{s}} \subseteq \mathcal{Q}_{s} \subseteq \mathcal{R}_{\eta} \subseteq \mathcal{Q}_{w} \subseteq \mathcal{R}_{\epsilon_{w}^{\prime}}$ induce a sequence of maps

$$
H_{1}\left(\mathcal{S}_{\epsilon_{s}}\right) \rightarrow H_{1}\left(\mathcal{Q}_{s}\right) \rightarrow H_{1}\left(\mathcal{S}_{\eta}\right) \rightarrow H_{1}\left(\mathcal{Q}_{w}\right) \rightarrow H_{1}\left(\mathcal{S}_{\epsilon_{w}^{\prime}}\right)
$$

where we have substituted $H_{1}(\mathcal{S})=H_{1}(\mathcal{R})$ using Theorem 3.1 and the fact that $H_{1}$ is the abelianization of $\pi_{1}$. The rank estimates now follow from linear algebra.

Knowing two "noisy" quasi-Rips complexes and the inclusion relating them yields true information about the (otherwise unobservable) shadow.

\section{5 k-Connectivity in $\mathbb{R}^{n}$}

Theorem 3.1 points to the broader question of whether higher-order topological data are preserved by the shadow projection map. Recall that a topological space is $k$ connected if the homotopy groups $\pi_{i}$ vanish for all $0 \leq i \leq k$. A map between topological spaces is $k$-connected if the induced homomorphisms on $\pi_{i}$ are isomorphisms for all $0 \leq i \leq k$.

We summarize the results of this section in Fig. 8.

Throughout this paper, we have ignored basepoint considerations in the description and computation of $\pi_{1}$. The following proposition excuses our laziness.

Proposition 5.1 For any set of points in $\mathbb{E}^{n}$, the map $p: \mathcal{R} \rightarrow \mathcal{S}$ is 0 -connected.

Proof Certainly $\pi_{0}(p)$ is surjective, since $p$ is surjective. The injectivity of $\pi_{0}(p)$ is a consequence of the following claim: If two Rips simplices $\sigma$ and $\tau$ have intersecting shadows, then $\sigma$ and $\tau$ belong to the same connected component of $\mathcal{R}$.

To prove the claim, suppose that $p(\sigma)$ and $p(\tau)$ intersect. By translation, we can suppose that $0 \in p(\sigma) \cap p(\tau)$. If $\left\{x_{i}\right\}$ and $\left\{y_{j}\right\}$ respectively denote the vertices of $\sigma$ and $\tau$, then

$$
\sum_{i} \lambda_{i} x_{i}=0=\sum_{j} \mu_{j} y_{j}
$$


Fig. 8 For which $(n, k)$ is the Rips projection map in $\mathbb{E}^{n}$ $k$-connected? The only unresolved case is $(3,2)$

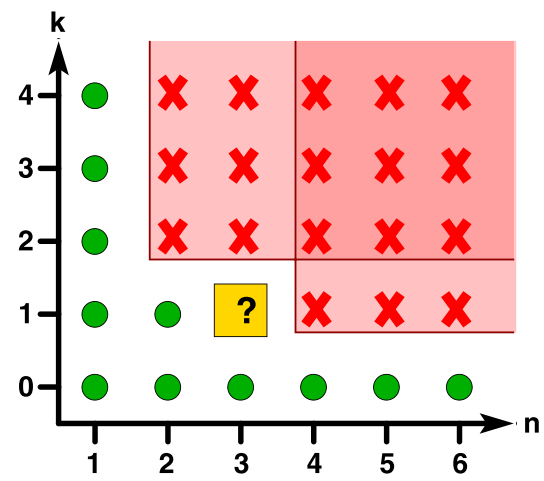

for suitable convex coefficients $\left\{\lambda_{i}\right\}$ and $\left\{\mu_{j}\right\}$. Then

$$
\begin{aligned}
\sum_{i, j} \lambda_{i} \mu_{j}\left|x_{i}-y_{j}\right|^{2} & =\sum_{i, j} \lambda_{i} \mu_{j}\left|x_{i}\right|^{2}-2 \sum_{i, j} \lambda_{i} \mu_{j}\left(x_{i} \cdot y_{j}\right)+\sum_{i, j} \lambda_{i} \mu_{j}\left|y_{j}\right|^{2} \\
& =\sum_{i} \lambda_{i}\left|x_{i}\right|^{2}-2 \sum_{i} \lambda_{i} x_{i} \cdot \sum_{j} \mu_{j} y_{j}+\sum_{j} \mu_{j}\left|y_{j}\right|^{2} \\
& =\sum_{i} \lambda_{i}\left|x_{i}\right|^{2}+\sum_{j} \mu_{j}\left|y_{j}\right|^{2},
\end{aligned}
$$

and similarly

$$
\begin{aligned}
\sum_{i, i^{\prime}} \lambda_{i} \lambda_{i^{\prime}}\left|x_{i}-x_{i^{\prime}}\right|^{2} & =2 \sum_{i} \lambda_{i}\left|x_{i}\right|^{2}, \\
\sum_{j, j^{\prime}} \mu_{j} \mu_{j^{\prime}}\left|y_{j}-y_{j^{\prime}}\right|^{2} & =2 \sum_{j} \mu_{j}\left|y_{j}\right|^{2} .
\end{aligned}
$$

Since every edge $x_{i} x_{i^{\prime}}$ and $y_{j} y_{j^{\prime}}$ has length at most 1 , the left-hand sides of these last equations have value at most 1 . Thus $\sum_{i} \lambda_{i}\left|x_{i}\right|^{2} \leq 1 / 2$ and $\sum_{j} \mu_{j}\left|y_{j}\right|^{2} \leq 1 / 2$. It follows that $\sum_{i, j} \lambda_{i} \mu_{j}\left|x_{i}-y_{j}\right|^{2} \leq(1 / 2)+(1 / 2)=1$, and so at least one edge $x_{i} y_{j}$ has length at most 1 .

Thus the simplices $\sigma, \tau$ are connected by an edge, as required.

Proposition 5.2 For any set of points in $\mathbb{E}^{1}$, the map $p: \mathcal{R} \rightarrow \mathcal{S}$ is a homotopy equivalence.

Proof Both $\mathcal{R}$ and $\mathcal{S}$ are homotopy equivalent to finite unions of closed intervals in $\mathbb{E}^{1}$, and therefore to finite sets of points. This is clear for $\mathcal{S}$. For $\mathcal{R}$, we note that $\mathcal{R}_{1}$ is equal to the Čech complex $\mathcal{C}_{1}$ in $\mathbb{E}^{1}$. Certainly the two complexes have the same 1-skeleton. Moreover, Helly's theorem implies that Čech complexes are flag completions in 1D: a collection of convex balls has nonempty intersection if all pairwise intersections are nonempty. Thus $\mathcal{R}_{1}=\mathcal{C}_{1}$. By the nerve theorem, this complex has the homotopy type of a union of closed intervals in $\mathbb{E}^{1}$. 
Since a 0-connected map between finite point sets is a homotopy equivalence, the same conclusion now holds for the 0 -connected map $p: \mathcal{R} \rightarrow \mathcal{S}$.

Proposition 5.3 There exists a configuration of points in $\mathbb{E}^{2}$ for which $p$ is not 2connected.

Proof Consider the vertices $r x_{1}, r x_{2}, r x_{3}, r x_{4}, r x_{5}, r x_{6}$ of a regular hexagon of radius $r$ centered at the origin. If $1 / 2<r \leq 1 / \sqrt{3}$, then only the three main diagonals are missing from $\mathcal{R}$. Thus $\mathcal{R}$ has the structure of a regular octahedron, and therefore the homotopy type of a 2 -sphere. On the other hand, $\mathcal{S}$ is just the hexagon itself (including interior) and is contractible.

The example of Proposition 5.3 extends to higher homotopy groups by constructing cross-polytopes, as in [9].

Proposition 5.4 There exists a configuration of points in $\mathbb{E}^{4}$ for which $p$ is not 1 connected.

Proof Consider the six points

$$
\left(r x_{1}, \epsilon x_{1}\right), \quad\left(r x_{2}, 0\right), \quad\left(r x_{3}, \epsilon x_{3}\right), \quad\left(r x_{4}, 0\right), \quad\left(r x_{5}, \epsilon x_{5}\right), \quad\left(r x_{6}, 0\right)
$$

in $\mathbb{E}^{4}$, in the notation of the previous proposition. Then $\mathcal{R}$ has the structure of a regular octahedron, but the map $p: \mathcal{R} \rightarrow \mathcal{S}$ identifies one pair of antipodal points (specifically, the centers of the two large triangles, 135 and 246). Thus $\mathcal{R}$ is simplyconnected, whereas $\pi_{1}(\mathcal{S})=\mathbb{Z}$.

We note that these counterexamples may be embedded in higher dimensions. Moreover, the example in Proposition 5.3 can be perturbed into general position without losing its effect, and the example in Proposition 5.4 can be put into general position by the dismal trick of replacing each vertex with a tiny cluster of vertices.

\section{Conclusion}

The relationship between a Rips complex and its projected shadow is extremely delicate, as evidenced by the universality result for quasi-Rips complexes (Theorem 4.1) and the lack of general $k$-connectivity in $\mathbb{R}^{n}$ (Sect. 5). These results act as a foil to Theorem 3.1: it is by no means a priori evident that a planar Rips complex should so faithfully capture its shadow.

We close with a few remarks and open questions.

(1) Are the cross-polytopes of Proposition 5.3 the only significant examples of higher homology in a (planar) Rips complex? If all generators of the homology $H_{k}(\mathcal{R})$ for $k>1$ could be classified into a few such "local" types, then, after a local surgery on $\mathcal{R}$ to eliminate higher homology, one could use the Euler characteristic combined with Theorem 3.1 as a means of quickly computing the number of holes in the shadow of a planar Rips complex. This method would have the advantage of being local and thus distributable. 
(2) Does the projection map preserve $\pi_{1}$ for a Rips complex of points in $\mathbb{R}^{3}$ ? Our proofs for the 2-d case rest on some technical lemmas whose extensions to 3-d would be neither easy to write nor enjoyable to read. A more principled approach would be desirable, but is perhaps not likely given that 1 -connectivity on $\mathbb{R}^{3}$ is a borderline case.

(3) What are the computational and algorithmic issues associated with determining the shadow of a (planar) Rips complex? See [6] for recent progress, including algorithms for test contractibility of cycles in a planar Rips complex and a positive lower bound on the diameter of a hole in the shadow.

\section{References}

1. Barrière, L., Fraigniaud, P., Narayanan, L.: Robust position-based routing in wireless ad hoc networks with unstable transmission ranges. In: Proc. Workshop on Discrete Algorithms and Methods for Mobile Computing and Communications (2001)

2. Björner, A.: Topological methods. In: Graham, R., Grötschel, M., Lovász, L. (eds.) Handbook of Combinatorics, 1819-1872. North-Holland, Amsterdam (1995)

3. Carlsson, G., Zomorodian, A., Collins, A., Guibas, L.: Persistence barcodes for shapes. Int. J. Shape Model. 11, 149-187 (2005)

4. Carlsson, E., Carlsson, G., de Silva, V.: An algebraic topological method for feature identification. Int. J. Comput. Geom. Appl. 16(4), 291-314 (2006)

5. Carlsson, G., Ishkhanov, T., de Silva, V., Zomorodian, A.: On the local behavior of spaces of natural images. Int. J. Comput. Vis. 76(1), 1-12 (2008)

6. Chambers, E., Erickson, J., Worah, P.: Testing contractibility in planar Rips complexes. In: Proc. Symp. on Comp. Geom. (SoCG), pp. 251-259 (2008)

7. Damian, M., Pandit, S., Pemmaraju, S.: Local approximation schemes for topology control. In: Proc. ACM Symp. on Prin. of Dist. Comput. (PODC), pp. 208-217 (2006)

8. de Silva, V., Carlsson, G.: Topological estimation using witness complexes. In: SPBG'04 Symposium on Point-Based Graphics, pp. 157-166 (2004)

9. de Silva, V., Ghrist, R.: Coordinate-free coverage in sensor networks with controlled boundaries via homology. Int. J. Robot. Res. 25(12), 1205-1222 (2006)

10. de Silva, V., Ghrist, R.: Coverage in sensor networks via persistent homology. Alg. Geom. Top. 7, 339-358 (2007)

11. Eckhoff, J.: Helly, radon, and carathéodory type theorems. In: Gruber, P.M., Wills, J.M. (eds.) Handbook of Convex Geometry. North-Holland, Amsterdam (1993), pp. 389-448

12. Fang, Q., Gao, J., Guibas, L.: Locating and bypassing routing holes in sensor networks. In: Proc. 23rd Conference of the IEEE Communications Society (InfoCom) (2004)

13. Gromov, M.: Hyperbolic groups. In: Essays in Group Theory. MSRI Publ., vol. 8. Springer, Berlin (1987)

14. Hatcher, A.: Algebraic Topology. Cambridge University Press, Cambridge (2002)

15. Haussman, J.-C.: On the Vietoris-Rips complexes and a cohomology theory for metric spaces. In: Prospects in Topology. Ann. of Math. Stud., vol. 138. Princeton Univ. Press, Princeton (1995), pp. 175-188

16. Kuhn, F., Wattenhofer, R., Zollinger, A.: Ad-hoc networks beyond unit disk graphs. Wirel. Netw. 14(5), 715-729 (2008)

17. Muhammad, A., Jadbabaie, A.: Dynamic coverage verification in mobile sensor networks via switched higher order Laplacians. In: Robotics: Science \& Systems (2007)

18. Niyogi, P., Smale, S., Weinberger, S.: Finding the homology of submanifolds with high confidence from random samples. Discrete Comput. Geom. 39(1), 419-441 (2008)

19. Vietoris, L.: Über den höheren Zusammenhang kompakter Räume und eine Klasse von zusammenhangstreuen Abbildungen. Math. Ann. 97, 454-472 (1927) 